\title{
Aportes desde la neurociencia, una perspectiva transformada para el aula
}

\section{Contributions from neuroscience, a transformed perspective for the classroom}

1 Verónica Adriana Freire Palacios

https://orcid.org/0000-0001-9493-6552. Universidad Técnica de Ambato, Facultad de Ciencias Humanas y de la Educación, Carrera de Educación Básica, Ambato, Ecuador va.freire@uta.edu.ec

2 Mentor Javier Sánchez Guerrero iD https://orcid.org/0000-0002-3762-7646 Universidad Técnica de Ambato, Facultad de Ciencias Humanas y de la Educación, Carrera de Pedagogía de la Actividad Física y Deportes, Ambato, Ecuador, jsanchez@uta.edu.ec

3 Willyams Rodrigo Castro Dávila (iD) https://orcid.org/0000-0002-2917-3461. Universidad Técnica de Ambato, Facultad de Ciencias Humanas y de la Educación, Carrera de Educación Básica, Ambato, Ecuador williamsrcastrod@uta.edu.ec

4 Jorge Luis Armijos Carrión Universidad Técnica de Machala, Facultad de Ciencias Sociales, Carrera de Pedagogía de las Ciencias Experimentales, Machala, Ecuador jlarmijos@utmachala.edu.ec

Artículo de Investigación Científica y Tecnológica Enviado: 24/12/2021

Revisado: $29 / 12 / 2021$

Aceptado: $12 / 01 / 2022$

Publicado:08/03/2023

DOI: https://doi.org/10.33262/concienciadigital.v6i1.4.2040

Freire Palacios, V. A., Sánchez Guerrero, M. J., Castro Dávila, W. R., \& Armijos Carrión, J. L. (2023). Aportes desde la neurociencia, una perspectiva transformada para el aula. ConcienciaDigital, 6(1.4), 918-930. https://doi.org/10.33262/concienciadigital.v6i1.4.2040

CONCIENCIA DIGITAL, es una Revista Multidisciplinar, Trimestral, que se publicará en soporte electrónico tiene como misión contribuir a la formación de profesionales competentes con visión humanística y crítica que sean capaces de exponer sus resultados investigativos y científicos en la misma medida que se promueva mediante su intervención cambios positivos en la sociedad. https://concienciadigital.org La revista es editada por la Editorial Ciencia Digital (Editorial de prestigio registrada en la Cámara Ecuatoriana de Libro con No de Afiliación 663) www.celibro.org.ec 
Palabras claves: neurociencia, neuroeducación, neurodidáctica, docente, aprendizaje

Keywords: neuroscience, neuroeducation, neurodidactics, teacher, learning
Resumen

Introducción: En la actualidad, el campo de las Neurociencias ha generado importantes avances enfatizando el estudio de los procesos cognitivos, dando lugar así al nacimiento de disciplinas en diversas ramas del conocimiento científico. Entre estas disciplinas destacan la neuroeducación y la neurodidáctica, cuyos aportes son de suma importancia para los docentes que buscan basar su práctica pedagógica en el funcionamiento y respuesta del cerebro en el aula. Objetivo: El objetivo de este artículo es realizar una amplia revisión bibliográfica de los últimos 5 años, en artículos de impacto de diferentes autores, para analizar las particularidades de las definiciones a partir del significado de la neurociencia y su relación con otras ciencias. Metodología: Se utilizaron métodos de investigación teóricos y empíricos, como analítico-sintético en la sistematización de la revisión exhaustiva de los principales fundamentos que sustentan este trabajo. Resultados: Se muestra un resumen de los diferentes artículos revisados, indicando a qué revista corresponden, señalando sus autores y el año de publicación. Conclusiones: Finalmente, se concluye sobre la importancia de estos aportes y cómo orientan el rol docente y las prácticas educativas para posibilitar el logro de aprendizajes significativos en el aula, por supuesto incluyendo los conceptos, preceptos y teoría revisados en este trabajo.

\section{Abstract}

Introduction: At present, the field of Neurosciences has generated significant progress emphasizing the study of cognitive processes, thus giving rise to the birth of disciplines in various branches of scientific knowledge. Among these disciplines, neuroeducation and neurodidactics stand out, and their contributions are of utmost importance for teachers who seek to base their pedagogical practice on how the brain works and responds in the classroom. Objective: The objective of this article is to carry out a broad literature review of the last 5 years, in articles of impact of different authors, to analyze the particularities of the definitions starting from the meaning of neuroscience and its relationship with other sciences. Methodology: Theoretical and empirical research methods were used, as analytical-synthetic in the systematization of the exhaustive review of the main foundations that support this work. Results: A summary of the different articles reviewed is shown, indicating 
which journal they correspond to, stating their authors and the year of publication. Conclusions: Finally, we conclude on the importance of these contributions and how they guide the teaching role and educational practices to enable the achievement of significant learning in the classroom, of course including the concepts, precepts and theory reviewed in this work.

\section{Introducción}

La neurociencia estudia el cerebro y permite comprender su funcionamiento, según Blakemore \& Frith (2007) afirman que las neurociencias constituyen un conjunto de saberes que se enfocan en estudiar la estructura y el funcionamiento del sistema nervioso, así como la interacción de los elementos del cerebro que dan origen a la conducta de los seres humanos (Cumpa, 2019). Cuando se habla de neurociencias se abarcan varias disciplinas relacionadas con el estudio del cerebro, el cual es un órgano tan complejo, que, a pesar del desarrollo y avance del hombre, no se ha podido crear una máquina similar al mismo.

Según Marueria "La neurociencia es la disciplina encargada de estudiar el cerebro y como éste da origen a la conducta y el aprendizaje" (p.267), las neurociencias se ocupan además de estudiar la plasticidad del sistema nervioso, de la importancia del ambiente en el aula, las bases de la motivación, la atención, las emociones y la memoria, como constituyentes esenciales del proceso de enseñanza-aprendizaje (Benavidez \& Flores, 2019); por lo tanto se comprende que la neuroeducación y la neurodidáctica son disciplinas que se encargan de la relación enseñanza-aprendizaje y cerebro.

El siguiente artículo se enfoca en un análisis de las aproximaciones conceptuales de la neurociencia y su relación con otras ciencias, para entender, así como el cerebro aprende y que es lo que necesita para su estimulación. La estructura de este artículo se llevó a cabo con una revisión documental especifica centrada en el tema, considerando publicaciones de revistas indexadas como Digitalia Hispana, Scielo, Redalyc, Redined, Refcale y diferentes bases de datos, bajo las siguientes palabras clave: neurociencias, inteligencia emocional, cerebro emocional, aprendizaje humano, neurodidáctica y neuroeducación.

En la última década del siglo XX conocida como la década del cerebro ha surgido una nueva corriente como son las neurociencias. Velásquez et al. (2009) mencionan que las neurociencias se refieren al estudio del sistema nervioso, desde diversos enfoques y a través de diferentes disciplinas, desde la Biología Molecular, la Fisiología, la Genética, la Psicología, entre otras (Benavidez \& Flores, 2019). Es así como Tacca et al. (2019), afirman que la neurociencia es calificada como un área multidisciplinar que indaga acerca 
de lo referente al cerebro y al sistema nervioso (constitución, funcionamiento, manifestación, evolución, etc.).

Mendoza et al. (2019) aseveran que las neurociencias se definen como aquellas investigaciones dirigidas a dilucidar la estructura y las funciones del sistema nervioso y el cerebro. Los adelantos de las tecnologías de la información propician una búsqueda científica que permite conocer con mayor profundidad funciones cerebrales superiores y complejas, como el lenguaje, la memoria y la atención; y cómo estos procesos se perfeccionan en las diferentes etapas de la vida.

En tal contexto, es importante mencionar que los últimos descubrimientos de la neurociencia han alcanzado otros campos como el marketing, la psicología, la educación, abriendo caminos para comprender de manera integral el funcionamiento del comportamiento humano y no solamente del cerebro y sus estructuras (Tacca et al., 2019). Por tal motivo, al mencionar que la neurociencia es multidisciplinaria surgen algunos campos de estudio que están reconocidos por la comunidad científica como los que definen: Blakemore \& Frith (2007) en su glosario de términos:

- Neuroanatomía. - Estudio de la estructura del cerebro.

- Neurobiología. - Estudio de la estructura y la función del cerebro.

- Neurología. - Diagnóstico y tratamiento clínico de pacientes con enfermedades o lesiones neurales.

- Neuropsicología. - Diagnóstico, estudio y tratamiento de personas que han sufrido lesiones cerebrales (Cumpa, 2019).

De acuerdo con Manes \& Niro (2015) definen otras ramas de estudio de la neurociencia que se relacionan con la ética, la educación y el marketing por mencionar algunas que también están ligadas directamente al comportamiento humano y que sin lugar a duda el cerebro juego un rol importante en las repuestas a los diferentes estímulos que proporciona el entorno, ya que el cerebro procesa la información para la vida en sociedad. Estas ramas son:

- Neuroeconomía. - Estudio de las bases neurales de los procesos cognitivos y emocionales para la toma de decisiones económicas.

- Neuroeducación. - Disciplina que tiene como objetivo el desarrollo de nuevos métodos de enseñanza y aprendizaje combinando pedagogía, neurobiología y ciencias cognitivas.

- Neuroética. - Reflexión sistemática y crítica sobre cuestiones fundamentales en los avances científicos del cerebro, como las implicancias filosóficas, sociales y legales.

- Neurofilosofía. - Estudio de la libertad de decisión de las personas, de su libre albedrío. 
- "Neuromarketing". - Los autores colocan este término entre comillas precisamente para cuestionar su carácter científico. Concluyen que no. Supuestamente, mediante este procedimiento se analizan las percepciones de los gustos de los consumidores para predecir su comportamiento de compra.

Considerando los antecedentes expuestos, existe una mirada amplia de que la neurociencia es una disciplina científica que abarca otras ciencias que permiten tener enfoques más claros de como el cerebro funciona y responde, y es así como nos enfocaremos en desarrollar los conceptos de la neuroeducación disciplina científica que aporta a entender como los docentes deben aplicarla para obtener mayor éxito en todos los procesos relacionados al aprendizaje.

Meza \& Moya (2020) indican que en las últimas décadas la Neuroeducación plantea analizar nuevas temáticas que permitirán el fortalecimiento de la educación, es decir potencializar el aprendizaje de los estudiantes mediante el estudio del cerebro y su funcionamiento, como también conocer que este órgano está constituido por neuronas capaces de regenerarse y de sufrir cambios en repuesta a estímulos, movimientos y actividades mentales, como también responder a diversas experiencias. No obstante, la neuroeducación con ayuda de la neurociencia intenta encontrar vías a través de las cuales poder aplicar en el aula los conocimientos que van apareciendo sobre el cerebro en relación con la emoción, la curiosidad y la atención ya que desempeñan un papel importante en el neurodesarrollo, están arraigadas biológicamente a nuestra naturaleza. Para que el estudiante aprenda, debe hacerlo en un ambiente cálido, donde se sienta respetado, apoyado y considerado como persona y no sentirse amenazado o maltratado.

Por su parte López (2014) afirma que la neuroeducación surge del traslape de tres campos disciplinares: la psicología, las neurociencias y la pedagogía, en otras palabras, el estudio de la mente, los fenómenos biológicos que explican el funcionamiento del cerebro y los procesos de formación que tienen lugar a través de la enseñanza y el aprendizaje, por consiguiente es de vital importancia que los docentes conozcan estas disciplinas y que sean parte de su formación para aplicar en el aula y que el aprendizaje sea significativo y holístico.

Desde una visión pedagógica, no se puede dejar de lado otra rama de la neurociencia que es la neurodidáctica y coinciden con Ocampo (2020) la Neurodidáctica es una disciplina científica naciente, que forma un segmento de la neurociencia y trabaja la optimización del aprendizaje basado en el desarrollo del cerebro; sin duda, todo aprendizaje está basado en el funcionamiento del cerebro y las estructuras neuronales. Por consiguiente, la Neurodidáctica es una disciplina pedagógica que tiene como objetivo diseñar estrategias metodológicas que optimicen el aprendizaje dentro del aula, cuyo objeto de estudio son los procesos y elementos 
de aprendizaje con base en el funcionamiento del cerebro y así contribuye a un mejor quehacer profesional de los docentes. Siguiendo a Ocampo (2020) las estrategias metodológicas están compuestas por un conjunto de procedimientos que promueven la indagación, análisis y construcción del conocimiento, mediante procesos lógicos y con el apoyo de estrategias operativas y socioemocionales.

Por otro lado, Fernández (2017) en su artículo refiere que la neurodidáctica es un concepto relativamente nuevo, que propone utilizar las aportaciones científicas sobre el cerebro en el aula. Su utilidad puede ser muy interesante dentro del plano de la inclusión, a pesar de que la atención debe ser individualizada y adaptada la neurodidáctica puede ser más efectiva para estudiantes que presentan NEE asociadas o no a una discapacidad y así generar un aprendizaje más inclusivo con igualdad de oportunidades y de condiciones.

Considerando los antecedentes expuestos, según Araya \& Espinoza (2020) es posible comprender que educar es cambiar las posibilidades del cerebro. Por tanto, magnifica la relevancia de la labor docente en el desarrollo efectivo del proceso de formación del estudiantado, por consiguiente, es menester la formación del docente que responda a la educación del siglo XXI, que se adapte y adopte nuevas formas de educar y comprender la individualidad y particularidad que presentan los cerebros de cada uno de sus estudiantes.

Por último es importante mencionar que la neurociencia, la neuroeducación y la neurodidáctica es una triada fundamental para comprender el funcionamiento del cerebro y como éste se relaciona con los procesos de enseñanza -aprendizaje; los docentes no sólo deben conocer estas ramas científicas sino que deben aplicarlas según las necesidades que presenten sus estudiantes garantizando el éxito de un aprendizaje para la vida con alumnos con capacidad de planificación, organización, toma de decisiones, capacidad de resolver problemas e inhibir impulsos funciones ejecutivas del cerebro que están presentes en cada actividad que se realice.

\section{Metodología}

La presente investigación responde a una revisión sistemática de tipo descriptivo, se utilizó métodos de investigación del nivel teórico y empírico, como analítico - sintético en la sistematización de los principales fundamentos que sustentan este trabajo, llevando a una revisión documental especifica centrada en el tema, considerando publicaciones de revistas indexadas como Digitalia Hispana, Scielo, Redalyc, Redined, Refcale y diferentes bases de datos, bajo las siguientes palabras clave: neurociencias, inteligencia emocional, cerebro emocional, aprendizaje humano, neurodidáctica y neuroeducación. En el proceso de la investigación, la búsqueda de la literatura fue realizada entre los meses octubre, noviembre y diciembre de 2021. 
Posteriormente, en el proceso de la revisión sistemática se utilizó una base de datos construida para el proceso de análisis temático de los artículos seleccionados, que se describen en la Tabla no. 1

\section{Resultados}

En la tabla 1, podemos resumir el resultado de la amplia revisión de la literatura concerniente al tema de nuestra investigación, artículos y trabajos que han procedido de importantes revistas a nivel de Latinoamérica y también de España.

\section{Tabla 1}

Artículos seleccionados para la síntesis temática

\begin{tabular}{|c|c|c|c|}
\hline TITULO & REVISTA & AUTOR/ES & AÑO \\
\hline $\begin{array}{l}\text { La importancia de las emociones } \\
\text { para la neurodidáctica }\end{array}$ & $\begin{array}{l}\text { Wimblu, Rev. } \\
\text { Estud. de Psicología }\end{array}$ & $\begin{array}{l}\text { Verónica Benavidez, } \\
\text { Ramón Flores, }\end{array}$ & 2019 \\
\hline $\begin{array}{l}\text { Estrategias Neurodidacticas, } \\
\text { satisfacción y rendimiento } \\
\text { académico en estudiantes } \\
\text { universitarios }\end{array}$ & $\begin{array}{l}\text { Cuadernos de } \\
\text { Investigación } \\
\text { Educativa }\end{array}$ & $\begin{array}{l}\text { Daniel Rubén Tacca } \\
\text { Huamán*1 } \\
\text { Ana Luisa Tacca } \\
\text { Huamán**2 } \\
\text { Miguel Ángel Alva } \\
\text { Rodríguez**3 }\end{array}$ & 2019 \\
\hline $\begin{array}{l}\text { Consideraciones sobre aportes de } \\
\text { las neurociencias al proceso } \\
\text { enseñanza - aprendizaje }\end{array}$ & Edusol & Guibo Silva, Adonis & 2020 \\
\hline $\begin{array}{l}\text { La libertad incorporada como } \\
\text { clave para la neuroeducación } \\
\text { moral }\end{array}$ & $\begin{array}{l}\text { Sophia, Colección de } \\
\text { Filosofía de la } \\
\text { Educación }\end{array}$ & $\begin{array}{l}\text { Gracia, Javier, \& } \\
\text { Gozálvez, Vicent }\end{array}$ & 2019 \\
\hline $\begin{array}{l}\text { Influencia de la Neuroeducación } \\
\text { en el rendimiento académico de } \\
\text { estudiantes universitarios del área } \\
\text { Química }\end{array}$ & Educere & $\begin{array}{l}\text { Ferrer, Kenna; Molero, } \\
\text { Lorelis; Leal, Anirelis; } \\
\text { Añez, Omaira; Araque, } \\
\text { María; Ávila, Ayari }\end{array}$ & 2020 \\
\hline $\begin{array}{l}\text { El resurgir de la neurociencia } \\
\text { ecuatoriana: La Revista } \\
\text { Ecuatoriana De Neurología }\end{array}$ & Neurol & Carlos Ramos-Galarza & 2017 \\
\hline $\begin{array}{l}\text { Usos y abusos del término } \\
\text { "neurociencias": una revisión } \\
\text { sistemática en revistas indexadas } \\
\text { Scielo }\end{array}$ & $\begin{array}{l}\text { Revista ConCiencia } \\
\text { EPG, }\end{array}$ & Moisés Cumpa-Valencia & 2019 \\
\hline $\begin{array}{l}\text { Neuroética: la institucionalización } \\
\text { de la ética en neurociencia }\end{array}$ & Rev. bioét. & $\begin{array}{l}\text { Amer Cavalheiro } \\
\text { Hamdan }\end{array}$ & 2017 \\
\hline $\begin{array}{l}\text { La Neurociencia: una postura } \\
\text { crítica frente al "boom" por la } \\
\text { "neuro" }\end{array}$ & CES Psicología & $\begin{array}{l}\text { Liliana Calderón } \\
\text { Delgado }\end{array}$ & 2017 \\
\hline
\end{tabular}




\section{Tabla 2}

Artículos seleccionados para la síntesis temática (continuación)

\begin{tabular}{|c|c|c|c|}
\hline TITULO & REVISTA & AUTOR/ES & AÑO \\
\hline $\begin{array}{l}\text { La neurociencia en la } \\
\text { formación inicial de } \\
\text { docentes }\end{array}$ & $\begin{array}{l}\text { CONRADO | Revista } \\
\text { pedagógica de la } \\
\text { Universidad de } \\
\text { Cienfuegos }\end{array}$ & $\begin{array}{l}\text { Jiménez Pérez, I. H., López } \\
\text { Rodríguez del Rey M. M., \& } \\
\text { Herrera González, D }\end{array}$ & 2019 \\
\hline $\begin{array}{l}\text { Neurodidáctica en el aula } \\
\text { creativa }\end{array}$ & CIVAE & María del Prado Camacho Alarcón & 2020 \\
\hline $\begin{array}{l}\text { Estrategia Neurodidáctica } \\
\text { para la formación de } \\
\text { investigadores sociales }\end{array}$ & Delectus & $\begin{array}{l}\text { OCAMPO EYZAGUIRRE, } \\
\text { DAVID }\end{array}$ & 2020 \\
\hline $\begin{array}{l}\text { Influencia del programa } \\
\text { neurodidáctica } \\
\text { "MATCERSPA" en el } \\
\text { aprendizaje de matemática } \\
\text { en estudiantes de } \\
\text { secundaria }\end{array}$ & $\begin{array}{l}\text { Revista CIENCIA Y } \\
\text { TECNOLOGÍA }\end{array}$ & $\begin{array}{l}\text { Nubia Ramírez Mamani de } \\
\text { Castañeda }\end{array}$ & 2020 \\
\hline $\begin{array}{l}\text { Modelo de formación } \\
\text { neuroeducativa para } \\
\text { docentes en la República } \\
\text { Dominicana }\end{array}$ & $\begin{array}{l}\text { Rev. Cubana Edu. } \\
\text { Superior }\end{array}$ & Yolanda Acta Caraballo & 2019 \\
\hline $\begin{array}{l}\text { Fundamentos } \\
\text { epistemológicos } \\
\text { transdisciplinares de } \\
\text { educación y neurociencia }\end{array}$ & Sophia & $\begin{array}{l}\text { Adela Fuentes Canosa, } \\
\text { Javier Collado Ruano }\end{array}$ & 2019 \\
\hline $\begin{array}{l}\text { Visiones desde la } \\
\text { neurociencia- } \\
\text { neurodidáctica para la } \\
\text { incorporación de las tic en } \\
\text { los escenarios educativos }\end{array}$ & $\begin{array}{l}\text { Revista de Ciencias } \\
\text { Sociales Ambos Mundos }\end{array}$ & $\begin{array}{l}\text { Julio Manuel Barroso Osuna1 Julio } \\
\text { Cabero Almenara2 Rubicelia } \\
\text { Valencia Ortiz3 }\end{array}$ & 2020 \\
\hline $\begin{array}{l}\text { TIC y neuroeducación } \\
\text { como recurso de } \\
\text { innovación en el proceso } \\
\text { de enseñanza y } \\
\text { aprendizaje }\end{array}$ & $\begin{array}{l}\text { ReHuSo: Revista de } \\
\text { Ciencias Humanísticas y } \\
\text { Sociales }\end{array}$ & $\begin{array}{l}\text { Luis Raúl Meza Mendoza } 1 \text { María } \\
\text { Elena Moya Martínez2 }\end{array}$ & 2020 \\
\hline $\begin{array}{l}\text { Neurodidáctica y } \\
\text { competencias emocionales } \\
\text { de estudiantes de } \\
\text { educación general básica }\end{array}$ & $\begin{array}{l}\text { CIENCIAMATRIA } \\
\text { Revista Interdisciplinaria } \\
\text { de Humanidades, } \\
\text { Educación, Ciencia y } \\
\text { Tecnología }\end{array}$ & $\begin{array}{l}\text { Aidee Monserrate Ramos García; } \\
\text { Esthela María San Andrés Laz }\end{array}$ & 2019 \\
\hline
\end{tabular}


Tabla 3

Artículos seleccionados para la síntesis temática (continuación)

\begin{tabular}{|c|c|c|c|}
\hline TITULO & REVISTA & AUTOR/ES & AÑO \\
\hline $\begin{array}{l}\text { Las neurociencias como } \\
\text { herramienta de } \\
\text { comunicación y desarrollo } \\
\text { de los procesos de } \\
\text { globalización }\end{array}$ & $\begin{array}{l}\text { Rev. Chilena. } \\
\text { Neuropsicología }\end{array}$ & $\begin{array}{l}\text { Nicolás Parra-Bolaños1*, Claudia } \\
\text { Estela Herrera-Cárdenas2, Albeiro } \\
\text { de Jesús Muñoz-Giraldo3 }\end{array}$ & 2017 \\
\hline $\begin{array}{l}\text { Aportes desde las } \\
\text { neurociencias para la } \\
\text { comprensión de los } \\
\text { procesos de aprendizaje en } \\
\text { los contextos educativos }\end{array}$ & $\begin{array}{l}\text { Propósitos y } \\
\text { Representaciones }\end{array}$ & $\begin{array}{l}\text { Sebastián C. Araya-Pizarro, Laura } \\
\text { Espinoza Pastén }\end{array}$ & 2020 \\
\hline $\begin{array}{l}\text { Neuro didáctica e } \\
\text { inclusión educativa }\end{array}$ & Publicaciones Didácticas & Fernández Palacio, Ana & 2017 \\
\hline $\begin{array}{l}\text { La gamificación como } \\
\text { herramienta en el trabajo } \\
\text { docente del orientador: } \\
\text { innovación en } \\
\text { asesoramiento vocacional } \\
\text { desde la neuro didáctica }\end{array}$ & $\begin{array}{l}\text { Revista Iberoamericana de } \\
\text { Educación }\end{array}$ & $\begin{array}{l}\text { Sonia Clementina Ferrer Planchart; } \\
\text { Mariana Fernández Reina; } \\
\text { Noirailith Daniela Polanco Padrón; } \\
\text { Maria Eugenia Montero Montero; } \\
\text { Estefany Elynett Caridad Ferrer }\end{array}$ & 2018 \\
\hline $\begin{array}{l}\text { Las neurociencias. una } \\
\text { visión de su aplicación en } \\
\text { la educación }\end{array}$ & Revista Órbita Pedagógica & $\begin{array}{l}\text { Angelita Azucena Falconi Tapia1 } \\
\text { Alexandra Lorena Alajo } \\
\text { Anchatuña2 Martha Cecilia Cueva3 } \\
\text { Rodolfo Matius Mendoza Poma4 } \\
\text { Santiago Fernando Ramírez } \\
\text { Jiménez5 Eliana Nathalie Palma } \\
\text { Corrales6 }\end{array}$ & 2017 \\
\hline $\begin{array}{l}\text { Neuro didáctica y } \\
\text { autorregulación del } \\
\text { aprendizaje, un camino de } \\
\text { la teoría a la práctica }\end{array}$ & $\begin{array}{l}\text { Revista Iberoamericana de } \\
\text { Educación }\end{array}$ & $\begin{array}{l}\text { Álvaro Federico Muchiut; Rocío } \\
\text { Beatriz Zapata; Alejandra Comba; } \\
\text { Martín Mari; } \\
\text { Noelia Torres; Jéssica Pellizardi; } \\
\text { Ana Paula Segovia }\end{array}$ & 2018 \\
\hline $\begin{array}{l}\text { Estrategias } \\
\text { Neurodidacticas en el } \\
\text { proceso enseñanza- } \\
\text { aprendizaje de educación } \\
\text { básica }\end{array}$ & $\begin{array}{l}\text { ReHuSo: Revista de } \\
\text { Ciencias Humanísticas y } \\
\text { Sociales }\end{array}$ & $\begin{array}{l}\text { Gina Carlota Briones Cedeño, } \\
\text { Jeovanny Benavides Bailón }\end{array}$ & 2021 \\
\hline $\begin{array}{l}\text { Neurociencia vs. } \\
\text { Neurodidáctica en la } \\
\text { evolución académica en la } \\
\text { educación superior }\end{array}$ & Revista Didasc@lia & $\begin{array}{l}\text { Carlos Emilio Paz Illescas1 Mónica } \\
\text { Patricia Acosta Gaibor2 Rosa } \\
\text { Erlinda Bustamante Cruz3 Carlos } \\
\text { Emilio Paz Sánchez4 }\end{array}$ & 2018 \\
\hline
\end{tabular}


Tabla 4

Artículos seleccionados para la síntesis temática (continuación)

\begin{tabular}{llll}
\hline \multicolumn{1}{c}{ TITULO } & \multicolumn{1}{c}{ REVISTA } & \multicolumn{1}{c}{ AUTOR/ES } & AÑO \\
\hline $\begin{array}{l}\text { La autorregulación del } \\
\text { aprendizaje de los } \\
\text { adolescentes y la neuro } \\
\text { didáctica }\end{array}$ & $\begin{array}{l}\text { Revista Ciencias } \\
\text { Pedagógicas e Innovación }\end{array}$ & $\begin{array}{l}\text { Freddy Tigrero Suárez, / Aníbal } \\
\text { Puya Lino, / Carlos José Apolinario } \\
\text { Tomalá, / Daniel Fabián Apolinario } \\
\text { Tomalá }\end{array}$ & 2020 \\
\hline $\begin{array}{l}\text { Percepciones de los } \\
\text { educadores sobre el papel } \\
\text { de la neurociencia en } \\
\text { educación resultados de } \\
\text { un estudio en España }\end{array}$ & Bordón Revista de & $\begin{array}{l}\text { María Teresa Martín } \\
\text { Aragoneses, Eva Expósito }\end{array}$ & 2021 \\
\hline $\begin{array}{l}\text { Importancia de la } \\
\text { Neurociencia en la }\end{array}$ & Revista Publicando & Anaya Nieto & \\
Educación & & Segundo Adolfo Bassante Jiménez & 2017 \\
\hline $\begin{array}{l}\text { La enseñanza-aprendizaje } \\
\text { en la educación superior: } \\
\text { aportaciones desde neuro } \\
\text { didáctica }\end{array}$ & Revista Didasc @lia & Emma Yolanda Mendoza Vargas1 & 2019 \\
& & Guadalupe Murillo Campuzano 2 & \\
\hline
\end{tabular}

Elaborado por: Los Investigadores.

\section{Conclusiones}

- A través de la revisión bibliográfica acerca de las neurociencias y su relación con diferentes ramas científicas, se logró comprender que el estudio del cerebro y su funcionamiento es un desafío para los docentes, ya que además de conocer, el reto está en aplicarla en el aula buscando las mejores didácticas y estrategias de enseñanza para que el cerebro de los estudiantes se active y esto se consigue con el quehacer diario dentro del aula empleando los principios de la neuroeducación y de la neuro didáctica.

- La revisión sistemática de diferentes artículos relacionados con la temática permitió concluir que ninguna rama de la neurociencia se desvincula del aprendizaje y no solo de un aprendizaje formal sino de un aprendizaje para la vida, tomando en cuenta que el ser humano es un ser biopsicosocial- cultural y religioso y que en cada una de estas dimensiones el cerebro responde y se activa para asimilar toda la información que está a su alrededor.

- Finalmente, el campo de las neurociencias aún falta por conocer, descubrir más, debido a que el cerebro siempre será un órgano que sorprenderá a los científicos, pues no nos estamos enfrentando a un simple conjunto de tejidos orgánicos, sino a un órgano complejo capaz de generar millones de conexiones y éstas permiten 
percibir, interpretar y aprender de maneras diferentes, haciéndonos a los seres humanos únicos e irrepetibles, con peculiaridades y similitudes en nuestra forma de pensar, actuar y sentir.

\section{Referencias Bibliográficas}

Araya, S., \& Espinoza, L. (2020). Aportes desde las neurociencias para la comprensión de los procesos de aprendizaje en los contextos educativos. Propósitos y Representaciones.

Benavidez, V., \& Flores, R. (2019). La importancia de las emociones para la neuro didáctica. Wimblu, Rev. Estud. de Psicología UCR, 25-53.

Blakemore, S., \& Frith, U. (2007). Cómo aprende el cerebro las claves para la educación. Sapiens: Revista Universitaria de Investigación, 133-136.

Cumpa, M. (2019). Usos y abusos del término "neurociencias": una revisión sistemática en revistas indexadas Scielo. ConCiencia EPG, 30-67.

Fernández, A. (2017). Neurodidáctica e inclusión educativa. Publicaciones Didácticas, 262-266.

López, V. (2014). Biología y educación: un nuevo punto de encuentro. Tendenci@s, 3238.

Manes, F., \& Niro, M. (2015). Usar el cerebro Conocer nuestra mente para vivir mejor. Barcelona España: PAIDÓS.

Mendoza, E., Murillo, G., \& Morales, A. (2019). LA ENSEÑANZA-APRENDIZAJE EN LA EDUCACIÓN SUPERIOR: APORTACIONES DESDE LA NEURODIDÁCTICA. Didasc@lia: Didáctica yEducación, 23-36.

Meza, L., \& Moya, M. (2020). TIC y neuroeducación como recurso de innovación en el proceso de enseñanza y aprendizaje. ReHuSo: Revista de Ciencias Humanísticas y Sociales, 85-96.

Ocampo, D. (2020). Estrategia Neurodidáctica para la formación de investigadores sociales. Delectus.

Tacca, D., Tacca, A., \& Alva, M. (2019). Estrategias neurodidácticas, satisfacción y rendimiento académico en estudiantes universitarios. Cuadernos de Investigación Educativa, 15-32. 


\section{DDigital}

\section{ISSN: 2600-5859}

Vol. 6 No. 1.4, pp. 918 - 930, marzo 2023

www.concienciadigital.org

Velásquez, B., Remolina, N., \& Calle, M. (2009). El cerebro que aprende. TABULA RASA, 329-347.

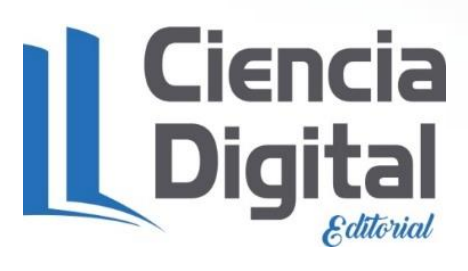


El artículo que se publica es de exclusiva responsabilidad de los autores y no necesariamente reflejan el pensamiento de la Revista Conciencia Digital.

\section{Ciencia}

El artículo queda en propiedad de la revista y, por tanto, su publicación parcial y/o total en otro medio tiene que ser autorizado por el director de la Revista Conciencia Digital.
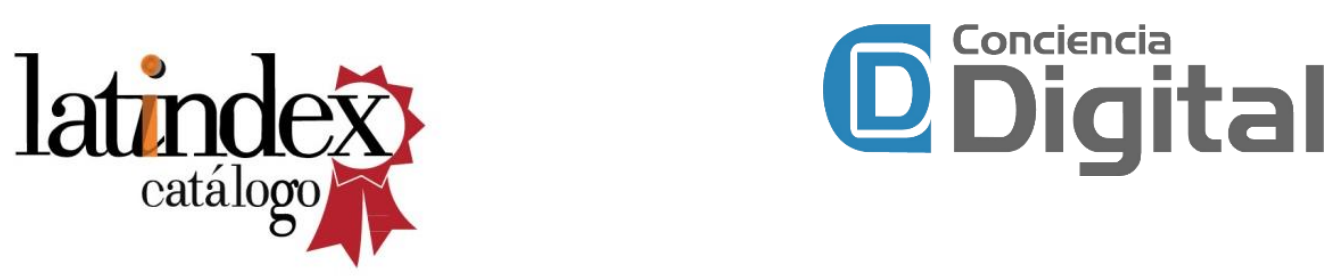

Indexaciones

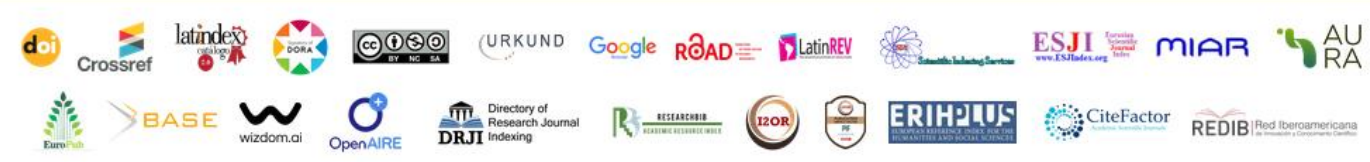

